Funded by COLCIENCIAS (Colombia) Grant: 6212009

\section{P2.062 OPTIMIZATIONS AND QUALITY ASSURANCE OF THE LABORATORY DIAGNOSIS AND TREATMENT OF SEXUALLY TRANSMITTED INFECTIONS IN BELARUS}

\author{
doi:10.1136/sextrans-2013-051184.0327
}

\begin{abstract}
10 Pankratov, "I Shimanskaya, ${ }^{2} \mathrm{~A}$ Lukyanau, ${ }^{3} \mathrm{M}$ Unemo, ${ }^{4} \mathrm{M}$ Domeika, Eastern European Network for Sexual Reproductive Health. ${ }^{1}$ Belarusian Medical Academy of Postgraduate Education, Minsk, Belarus; '2Belarusian State Medical University, Minsk, Belarus; ${ }^{3}$ WHO Collaborating Centre for Gonorrhoea and other STIS, Örebro, Sweden; ${ }^{4}$ Department of Control and Prevention of Communicable Diseases, Uppsala County Council, Uppsala, Sweden
\end{abstract}

Background In 2007-2008, a questionnaire-based study evaluated the quality of the 316 State laboratory services that were engaged in diagnosis of STIs in Belarus. This comprehensive survey clearly demonstrated that many of the tests and testing algorithms used in the laboratory diagnosis were inappropriate and not in accordance with international evidence-based recommendations.

Methods STI specialists from Belarus actively participated in the development of Eastern European consensus guidelines for the diagnosis of several STIs; an international collaborative work by the Eastern European Network for Sexual and Reproductive Health (EE SRH).

Results The international evidence-based guidelines developed by the EE SRH have subsequently been adapted to national conditions and legalised by the Ministry of Health of Belarus as the national standard for laboratory diagnosis of STIs. Briefly, antibody testing for diagnosis of genital Chlamydia trachomatis and Trichomonas vaginalis infections has been abandoned. Internationally validated nucleic acid amplification tests (NAATs) have been strongly promoted and also introduced for diagnosis of several STIs. Diagnosis of Mycoplasma genitalium using NAATs was initiated and routine screening and/or testing for Ureaplasma urealyticum, Mycoplasma hominis, Gardnerella vaginalis and Mobiluncus spp. was excluded from the recommendations supported by the State. Laboratory specialists from the 11 laboratories of the dermatovenereological dispensaries were trained in diagnostics using NAATs and laboratories supplied by the necessary equipment and reagents for NAAT diagnostics. The cultivation of Neisseria gonorrhoeae has been optimised and gonococcal antimicrobial resistance surveillance has been established. Finally, evidence-based national STI clinical protocols, including treatment recommendations, have been elaborated and legalised by the Ministry of Health of Belarus.

Conclusion The international EE SRH collaborative project has significantly improved the quality of the STI diagnostics and treatment in Belarus. A new EE SRH project is planned for Belarus, aiming to monitor and evaluate the implementation of the current developments.

\section{P2.063 VALIDATION OF COPAN ENAT, A MOLECULAR TRANSPORT MEDIUM, FOR THE COLLECTION AND PRESERVATION OF URINE SPECIMENS FOR THE DETECTION OF STI INFECTIONS WITH THE SEEGENE ANYPLEX II STI-7 V1.1 ASSAY}

doi:10.1136/sextrans-2013-051184.0328

S Razeti. Arrow Diagnostics Srl, Genoa, Italy

Backgrounds Urine is used for screening STI infections with molecular assays. Copan developed the eNat, a molecular medium that preserves and stabilises nucleic acid (NC), for collection, and storage of clinical specimens for microbial detection by real-time PCR. Seegene uses dry container (DC) for urine collection for detection of urogenital pathogens with the Anyplex II STI-7 (STI7).

Study objective was to validate the eNat for nucleic acid preservation in urines for STDs detection with the STI7 assays.
Methods In this study, 80 urines, collected in DC from patients attending a Milan STD clinic. Urines were tested as per current method and after adding urine to $1 \mathrm{ml} e \mathrm{Nat}$. To find the urine volume with same sensitivity as urine in DC, 1, 2, and $3 \mathrm{ml}$ urine in 1 $\mathrm{ml}$ eNAT were tested. After vortexing the eNAT samples, NC was extracted from 350ul with the Automated Purification Systems (NIMBUS IVD) and eluted in 100ul buffer. Purified NCs were tested with the with the Seegene STI7 assay.

Results In the 80 urine samples tested, 43 negative and 37 positive were detected with DC, while $1 \mathrm{ml}, 2 \mathrm{ml}$ and $3 \mathrm{ml}$ urine in eNAT detected 45.40 .40 negative or partial negative $(1,2,3)$ and 35.40 .40 positive $(1,2,3)$ respectively. More co-infections were detected with eNAT $3 \mathrm{ml}$. Loss of sensitivity with $1 \mathrm{ml}$ eNAT and inhibition with DC versus $3 \mathrm{ml}$ in eNAT was detected in 7 samples.

Conclusions Good agreement was found between Copan eNat-3 ml urine and urine in DC for the detection of 7 STI with the Seegene assay. Copan eNAT, is available in leak proof tube, easy to transport-store urines, prevents bacterial overgrowth, stabilises NC at RT and is compatible with the STI7 assay.

\section{P2.064 COMPARISON OF URINE COLLECTED IN DRY CONTAINER TO URINE COLLECTED, TRANSPORTED AND PRESERVED IN THE COPAN URISWAB FOR THE DETECTION OF STDS WITH THE SEEPLEX STD6 ACE ASSAY}

doi:10.1136/sextrans-2013-051184.0329

A Archenti. ASL Milano - Prevention Laboratory, Milan, Italy

Backgrounds Molecular urine devices are not compatible for all molecular assays and are not good bacteria culture. Copan produces the UriSwab (US), a LBM device used with the WASP automation. It's a leak-proof screw-cap tube with 3 treated sponges on a plastic stick to absorb and retain urine during transport and prevent bacterial overgrowth. UriSwab can be used for urine self-collection for STD screening by culture and molecular assays. Urine collected in dry container (DC) were compared to US for detection of Trichomonas vaginalis (TV), Mycoplasma hominis (MH), Mycoplasma genitalium (MG), Chlamydia trachomatis (CT), Neisseria gonorrhoeae (NG) and Ureaplasma urealyticum (UU).

Methods Duplicate urines were collected to-date from 153 patients attending a Milan STD clinic. One urine was collected in DC and another in US. For the DC, $5 \mathrm{ml}$ urine was placed in a tube, and both, DC tube and US were centrifuged at $3000 \mathrm{~g} / 20 \mathrm{~min}$. After discarding the supernatant, the cell pellets were eluted in PBS and nucleic acid was extracted with the OIAamp DNA Mini kit (Qiagen). 3 ul purified sample was tested with the Seeplex ${ }^{\circledR}$ STD6 ACE assay (Seegene Inc)

Results In the 153 urine, DC and US had 90 negative and 52 positive concordant $(91.25 \%)$ and 9 discordant $(9.75 \%)$ results; positive included $10 \mathrm{CT}, 11 \mathrm{MH}, 8 \mathrm{UU}, 5 \mathrm{NG}$ and $3 \mathrm{MG}$. In the discordant, DC had 3 positive missed by US while US had 4 positive missed by DC. No inhibition or TV was detected, the study is-ongoing. Conclusions Good agreement was found between the Copan US and the DC for storing urines for STIs with the Seeplex ${ }^{\circledR}$ STD6 ACE. The US is leak-proof, easy-to-transport, store urines for STIs with molecular assays, prevents overgrowth, stabilises bacteria for culture and facilitates self-collection for STI screening.

\section{P2.065 PRELIMINARY EVALUATION OF A COMMERCIALLY AVAILABLE IMMUNOBLOTTING METHOD WITH TREPONEMA PALLIDUM RECOMBINANT ANTIGENS FOR SEROLOGICAL DIAGNOSIS OF SYPHILIS}

doi:10.1136/sextrans-2013-051184.0330

A Pierro, L Bragaglia, A Moroni, M Landini, V Sambri. Unit of Clinical Microbiology, St. Orsola-Malpighi University Hospital, Bologna, Italy 